\title{
PRAGMATICS DRIVEN LAND COVER SERVICE COMPOSITION UTILIZING BEHAVIOR-INTENTION MODEL
}

\author{
Hao Wu ${ }^{\text {a,* }}$ Jun Chen ${ }^{\text {a }}$, Huaqiao Xing ${ }^{\text {b }}$, Songnian $\mathrm{Li}^{\text {c }}$, Juju Hu ${ }^{\text {d }}$ \\ ${ }^{a}$ National Geomatics Center of China, Beijing 100830, China - (wuhao, chenjun)@nsdi.gov.cn \\ ${ }^{\mathrm{b}}$ Geoscience and Surveying Engineering College, China University of Mining and Technology (Beijing), Beijing 100083, China - \\ xinghuaqiao@126.com \\ ${ }^{c}$ Department of Civil Engineering, Ryerson University, Toronto, Ontario M5B 2K3, Canada - snli@ ryerson.ca \\ ${ }^{\mathrm{d}}$ School of Environment Science and Spatial Informatics, China University of Mining and Technology, Xuzhou, 221116, China \\ - hujuju@126.com
}

Commission VI, WG VI/4

KEY WORDS: Global land cover; Pragmatics web; Service Composition; Behavior-Intention

\begin{abstract}
:
Web service composition is one of the key issues to develop a global land cover (GLC) information service portal. Aiming at the defect that traditional syntax and semantic service compositionare difficult to take pragmatic information into account, the paper firstly analyses three tiers of web service language and their succession relations, discusses the conceptual model of pragmatic web service, and proposes the idea of pragmatics-oriented adaptive composition method based on the analysis of some examples. On this basis it puts forward the pragmatic web service model based on Behavior-Intention through presetting and expression of service usability, users' intention, and other pragmatic information, develops the on-demand assembly method based on the agent theory and matching and reconstruction method on heterogeneous message, solves the key technological issue of algorithm applicability and heterogeneous message transformation in the process of covering web service composition on the ground, applies these methods into service combination, puts forward the pragmatic driven service composition method based on behavior-intention model, and effectively settles the issue of coordination and interaction of composite service invocation.
\end{abstract}

\section{INTRODUCTION}

The significance of global land cover (GLC) information has been recognized by many fields, such as global environmental change studies, biodiversity monitoring, earth system modelling and many other societal benefits areas (Herold et al., 2008; Verburg et al., 2011; Hansen and Loveland, 2012;Chen et al., 2013a;Gong et al., 2013).To facilitate the application of GLC information, many groups and organizations have published their data and provide some online processing tools in form of web service (Chen et al., 2011; Townshend et al., 2012). In September 2014, China donated their $30 \mathrm{~m}$ resolution GLC data to UN and developed the GLC information service portal, which have provided browsing and downloading services. However, in order to achieve the purpose of totally online processing and service computation, there are still many difficulties remains.

One of these problems is how to combine appropriate data and processing services to compose a service chain automatically. For example, to make the change detection of water class based on bi-temporal images, we have to classify the images firstly, and then use change detection algorithm to obtain the results. There are so many image classification algorithms (Xian et al., 2010; Feranec et al., 2007; Chen et al., 2012; Chen et al., 2013b), such as maximum likelihood classification (MLC), support vector machine (SVM) based classification, and decision tree classification (DTC), etc. But it is known to all that different classification algorithms may have different application scopes, which are decided by the temporal, spatial and spectral resolution of the image to be classified. So, when we encapsulate the algorithms into web service, we may have to add some extra information to let computer know the adaptation of different services.
To improve the situations of web service composition and computation, some researchers have made a lot of efforts to the semantic web (Chen et al., 2011; Fukatsu et al., 2011; Yue et al., 2004;Zhao et al., 2012;), and tried to achieve the dynamic integration of web service. Zhao(2012) considered that service composition can be viewed as a process of geospatial knowledge transformation, and present a three-phase approach to geospatial knowledge transformation for service chain. Cruz and Antonio(2012) use semantic descriptions of geospatial data quality requirements in a rule-based form, and improve the accuracy of the situations of nonconformities with geo-data quality that may occur during the execution of the Web Service composition. Yue(2012) proposes a workflow-based approach for discovery of complex geospatial features that uses spatial semantics and thematic semantics of feature types.

But the semantic web mainly focuses on description of parameters and interfaces of web service using the knowledge in different research domains, butrarely take account of the intention of web service consumers and the variable processing context of a work-flow. This may confuse the consumers when they have to choose the most appropriate web service from multiple ones which have the same meanings in parameters and interface. So it is clearly to find that, we must improve the current web service description method, if we could select the most applicable web service to fill the consumers' need.

This paper proposed a new service composition method from the view of pragmatics. Firstly we analyzed the essential ingredients and relations among the syntax, semantic, pragmatics level of web service from the viewpoint of linguistics, and then proposed a conceptual model of pragmatics web service by discussing on some examples. Secondly, we revealed that the abilities of the web service itself (Behavior) 
and intentions of web service consumers (Intention) are two aspects of pragmatics description for web services. Based on the formalized description of the Behavior and Intention, we extended the formal web service description model, and proposed a Behavior-Intention model to represent the applicability of the web service and the constraints of consumers' intention. Finally, we presented an integration method for the dynamic composition of land cover web services based on the matching of request parameters and the ingredients of Behavior-Intention model. The methods proposed by this paper may improve the current web service description model by adding pragmatics information, and be helpful for the dynamic composition of web services.

\section{BACKGROUND AND RESEARCH IDEA}

\subsection{Three tiers of web service language}

Web service language is an important tool for the expression service to achieve details and also the core basis of service integration at the same time (Sun et al., 2012; Cruz et al., 2012;Sangers et al., 2013). Traditional web service language includes two tiers of syntax and semantics, of which the service language at the syntax tier mainly provides definitions of functional interface, parameter type, message format and other contents, while the service language at the semantic tier emphasizes participants' sharing of the service attribute, interface, parameter and other relevant knowledge. However, they have always overlooked the expression and description of the context knowledge and language environment and other pragmatic information. In fact, pragmatic information has reflected the partial conceptual intention of certain service's participants or business procedure, and has important influences on control and optimization of service integration.

The concept of pragmatics was put forward by Charles Morris in 1930s firstly. He thought that linguistics includes three parts: namely syntax, semantics and pragmatics, of which syntax expresses the structure of symbols, semantics expresses the implication of symbols, and pragmatics expresses the context knowledge and language environment of symbols. Prosperous development of pragmatic web service has provided a new solving idea for the issue of methodology of service integration. However, the emergency of pragmatic web service is not to replace the original syntax and semantic service language. On the contrary, pragmatic service language must be based on syntax and semantic service language, which is inheritance and development of traditional service language. Their tasks are clear and they cooperate at the same time, and they have commonly constituted the language system of web service. From the perspective of understanding and using of web service language, the paper divides it into three tiers of syntax, semantics and pragmatics, and has respectively analyzed their language features and realization ways. The tier structure is as shown in Figure1.

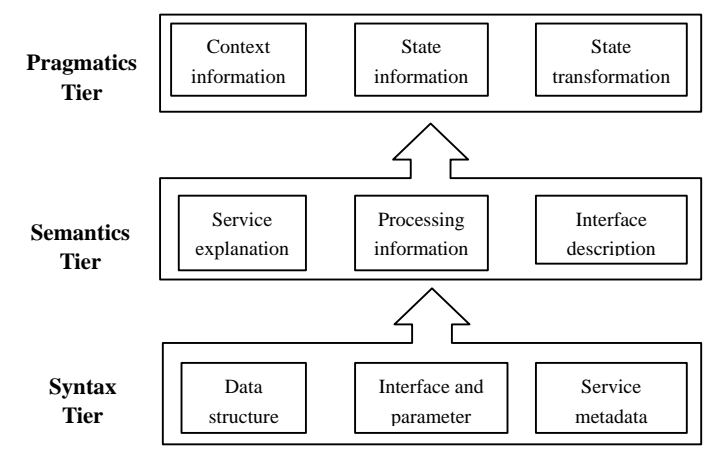

Figure 1.Main ingredients and relations of three tiers

1) Service language at the syntax tier achieves syntax unification of service mainly through the definitions of parameter format and message structure, such as solving the syntax heterogeneous issue described by the service through WSDL (Castronova et al., 2013). UDDI can support service searching based on key words. In the process of service invocation, the failure message won't be returned if the syntax requirement of the service is satisfied. However, it cannot guarantee that the returned message has actual meaning.

2) The service language at the semantic tier mainly uses domain ontology to label the semantics for the attribute and operation of the service (Farnaghi et al.,2013), and achieves intelligent service release, discovery and combination by sharing and reasoning of relevant knowledge of the service. Nevertheless, it only focuses on objective description of public information of the service (such as information interpretation and execution, and invocation and projection), and has overlooked the connotative and non-shared private knowledge of the service itself. In other words, semantic service language has offered objective semantic implication to the service users, but it's difficult to consider users' selection and procedure matching requirement for the service at the same time.

3) Established on the basis of semantic description, the service language at the pragmatic tier includes two parts: one is to describe context knowledge of the service, such as the issue of service applicability and how to use the service for users; second is to reflect the change of context knowledge through the description and transferring of the status, such as the conditions that need to be met before the service execution and how to handle the returned message after completion of the service execution.

\subsection{Conceptual model of pragmatic web service}

As previously mentioned, it becomes an irresistible trend for the development of web service from syntax web service and semantic web service to pragmatic web service. Compared with syntax and semantic web service, pragmatic web service has 3 basic features as below (Chen et al., 2008; Zhai et al., 2008):

1) Users first. Pragmatic web service emphasizes the description of the service context, so the information of the service consumers need to be added on the basis of existing service description, so as to make all service activities take the service consumers' requirements into full consideration.

2) Understanding prior to offering. Pragmatic web service requires us to be able to describe, use and understand the context information of the data and method before we offer the data and method. 
3) Interaction prior to expression. It refers to the extension of details and standards of the service interaction based on the semantic information against specific business logic.

Aiming at the basic features of pragmatic web service and combining three tiers of web service language, here we can use the classification concept to express the continuing relation among them and give the conceptual model of pragmatic web, as shown in Figure 2. Used for the modelling of the information world, the conceptual model is the first abstraction from the real world to the information world, and it has a strong ability in semantic expression and can express knowledge conveniently and directly that is involved in application. On the other hand, it also has the advantage of being simple, clear and easy for users to understand. In general, we can divide pragmatic web service into 4 kinds of basic concept, namely Who (service provider's information), What (service function and parameter), How (service semantic information), and Why (service context information). The specific contents are as below:

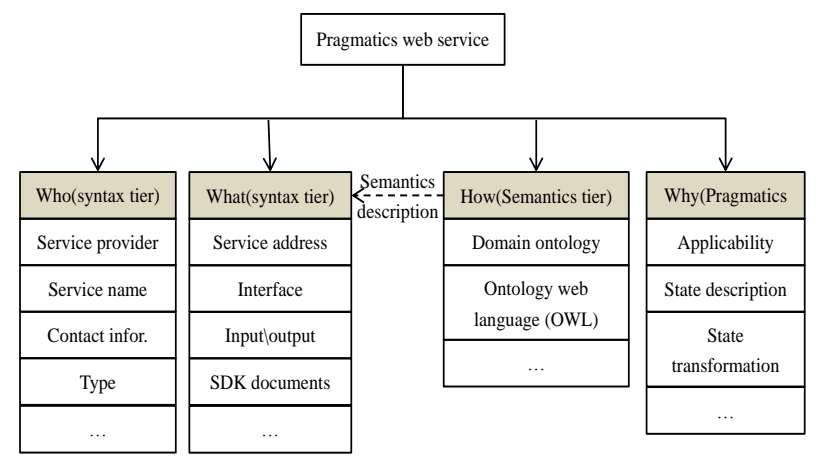

Figure 2. Conceptual model of pragmatics web service

1) Who refers to the entity information of the service provider, belongs to the service language at the syntax tier, and includes the service name, unit, contact information, and classification and so on.

2) What refers to the description information of the service realizing technology and belongs to the service language at the syntax tier, and it includes the service access point, functional interface, inputloutput parameter, and construction and standard and other technological information.

3) How refers to the semantic description information of the service realizing technology and belongs to the service language at the semantic tier. It labels the semantics to the service's basic components through domain ontology and ontology description language, so as to achieve the sharing and interaction of the service information.

4) Why refers to the context information of the service interaction and belongs to the service language at the pragmatic tier. It promotes dynamics of interaction between the service provider and the user through the introduction of the service users' conceptual intention, and includes service applicability, status description information, and status transfer information and so on.

\section{PRAGMATIC PRESETTING AND MODELING}

One of the important tasks of pragmatic web service integration is to harmonize the status information of various entities in the process of service interaction, which includes the entity's status description, and status transfer information relating to the composition context 2.1 and 2.2 have illustrated relevant entities of ground coverage information service operation in detail from the view of syntax and semantics. This section mainly discusses how to define hidden background knowledge in the process of service interaction from the perspective of pragmatics, so as to enable both parties of the interaction to understand the other party's conceptual intention more properly and correctly to achieve effective interaction.

\subsection{Essential content of pragmatic pre-setting}

Pragmatic ambiguity is the main reason that causes web service interaction failure. Here, we pre-set the pragmatic knowledge of each atomic service in a specific context by the way of pragmatic pre-setting to be used for describing the context information between the service provider and the user, so as to enhance both interaction parties' common understanding of the background knowledge. As mentioned previously, for the ground coverage information service, the issue of pragmatic ambiguity is mainly reflected in two aspects of the service applicability and the returned message type. Aiming at this status quo, we define the pragmatic pre-setting content of web service respectively from two perspectives of service behavior and user's intention, and establish the pragmatic sharing mechanism for both parties of service interaction.

3.1.1 Service behavior: Service behavior refers to the ability of atomic service to transform the operational entity according to certain target, and includes service function, applicability, direction for use, etc. It is the objective description of the entity status information in the process of service interaction. Among them, service function can be defined by the initial status and target status of the operational entity, applicability can use the relation between the initial status' attribute information and the applicability evaluation indicator for restriction, and direction for use is to enable the users to clearly understand how to use the message content returned by the service, is closely related to the attribute integration of the target status and can be described in the way of attribution expansion. In conclusion, we can use a six-tuple to define the service behavior of the atomic service.

Definition 1:Servicebehaviorofatomic service: $W_{\text {behavior }}=<R_{o}, A, R_{a}, I, O, R_{t}>$

Where $R_{o}$ : the initial statusof the operational entity.

$A$ : applicability evaluation indicator integration can be expanded according to concrete service types. For example, the applicability indicator of image classification service includes image spectral resolution, space resolution, sensor type and so on.

$R_{a}$ : the applicability relation integration between the attribute information of the operational entity and the evaluation indicator, $R_{a} \rightarrow R_{o} \times A$ indicates the applicability relation between the output parameter and the evaluation indicator. For $\forall r a \in R_{a}, \forall a \in A$ and $\forall r \in R_{o}$ (the attribute of $R_{o}$ ), if $\exists \mathrm{ra}(\mathrm{a})=\mathrm{r}$, it indicates the existence of applicability relation between the attribute and the evaluation indicator.

$I$ : The limited integration of the input information of Web service.

$O$ : The limited integration of the output information of Web service.

$R_{t}$ : The target status of the operational entity. Besides public attribute information, it still includes output type and other information.

Table 1 shows the service behavior of certain image classification algorithm. 


\begin{tabular}{|c|c|}
\hline$R_{O}$ & Remote sensing image \\
\hline$A$ & $\begin{array}{c}\text { High spatial resolution; Low spectrum } \\
\text { resolution }\end{array}$ \\
\hline$R_{a}$ & $\begin{array}{c}\text { Spatial resolution } \leq 30 \mathrm{~m} ; 1.2 \mathrm{mu} \leq \text { Spectral } \\
\text { resolution } \leq 2.5 \mathrm{mu}\end{array}$ \\
\hline$I$ & Threshold value for classification \\
\hline$O$ & Pixel amount \\
\cline { 2 - 2 } & Pixels percent \\
\hline$R_{t}$ & Classification image \\
\hline
\end{tabular}

Table1 Example for service behavior of a certain classification algorithm

3.1.2 User intention: In order to depict the process that the users expect certain atomic service to be able to change from the initial status to the target status through certain status switching in the specified input integration situation. It has strong independence and cannot be divided into several child intentions. Here, we define it through a tetrad.

Definition2 the service users' conceptual intention: $\mathrm{WS}_{\text {intention }}=<\mathrm{R}_{\mathrm{o}}{ }^{\prime}, \mathrm{I}^{\prime}, \mathrm{O}^{\prime}, \mathrm{R}_{\mathrm{t}}{ }^{\prime}>$

Where $\mathrm{R}_{\mathrm{o}}{ }^{\prime}$ : the initial status of the operational entity.

$\mathrm{I}^{\prime}$ : Limited integration of the input parameter, which can be provided in the process of users' intention realization.

$\mathrm{O}^{\prime}$ : Limited integration of the output parameter, which must be provided in the process of users' intention realization.

$\mathrm{R}_{\mathrm{t}}{ }^{\prime}$ : The target status of the operational entity, including output type and other information, after the realization of users' intention.

Table 2 shows the service intention of imagine classification algorithm.

\begin{tabular}{|c|c|}
\hline$R_{o}{ }^{\prime}$ & TM image \\
\hline$I^{\prime}$ & Threshold value for classification \\
\hline$O^{\prime}$ & Pixel amount \\
\hline$R_{t}{ }^{\prime}$ & Classification image \\
\hline
\end{tabular}

Table 2 Example for service intention of a certain classification algorithm

3.1.3 Pragmatic pre-setting principle: Pragmatic presetting of the service is conducive to the sharing and interaction of context information between the service provider and the user. What's worth pointing out is that the pragmatic information of different kinds of web service is not the same. To guarantee the accuracy of the pragmatic information, we should abide by the two principles as below in the process of pragmatic pre-setting:

1) The principle of appropriateness: Pragmatic appropriateness refers to whether pragmatics appropriateness has real meaning in the specified context. It is the prerequisite to guarantee the effectiveness of pragmatic pre-setting. For example, the service requester makes a request of "classifying the image", and whether this request is appropriate owns a series of pragmatic pre-setting as the prerequisite, like the acceptor must be the image classification service, this classification algorithm doesn't need the sample data of the training area. If the functional service of other types is requested or the classification algorithm needs to input the sample data of the training area, then this request is meaningless. Therefore, pre-setting pragmatic appropriateness is helpful for the service user to make the request correctly and understand the usage of the service accurately.
2) The principle of mutual understanding: The pre-setting content of pragmatics must be known by both parties of interaction and gain both parties' recognition. Here, mutual understanding of pragmatics is often closely combined with the context, namely, we can clearly define it in the users' intention and also input in the service request in a hidden way, for example, we can indicate the service user through Http:// 192.168.1.1/ ArcGIS/ WMS?Request=GetFeatureInfothat the returned message which the service users expect to get is the message type that the platform of ArcGIS can analyze.

\subsection{Contract expression of pragmatic presupposition}

In terms of the essence, the content of pragmatic presupposition is a multiparty contract. In order to make the pragmatic information have readability and computability, the presupposition content needs to be expressed in contract. Contract is a group of rules that describe service pragmatics, and it has provided hidden details of web service and the conceptual expression of the users' intention. As to atomic service, the contract content of the service behavior has been determined when the service is created. Thus, static language (such as OWL) can be adopted to express it. Because the service requester's usage intention is sent in real time by HTTP protocol, uniform SOAP message can be defined (simple object access protocol). Through analyzing the service request, the service requester's conceptual intention can be gained.

Because there is no internationally unified standard of pragmatic web service description currently, the paper adopts OWL to express the service behavior and takes it as the component of pragmatic web service description to bind with atomic service. Figure 4 has given the basic framework of service behavior contract expression.

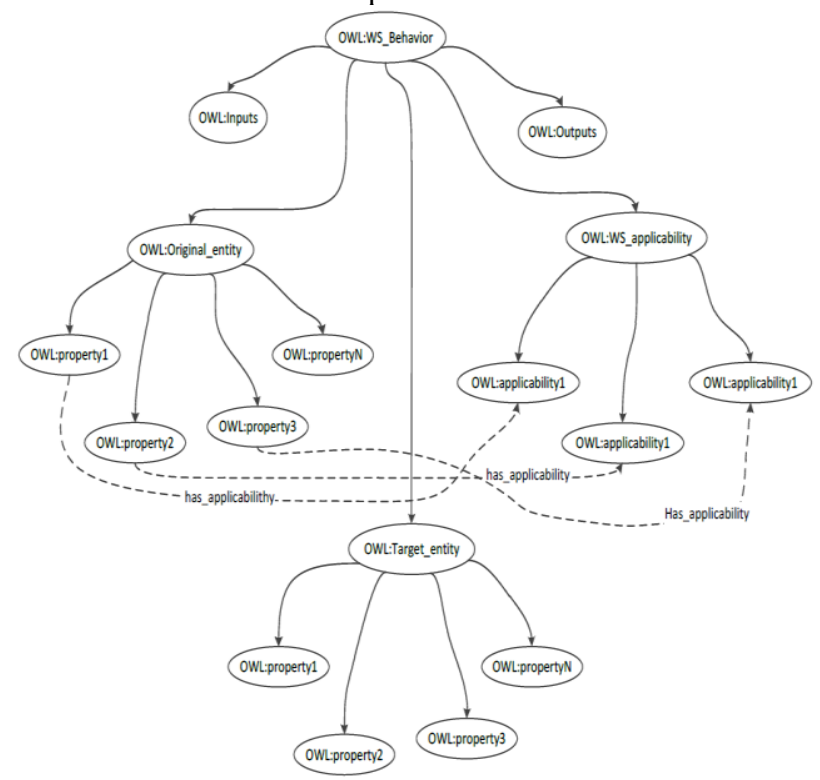

Figure. 4 Framework for expression service behavior

As mentioned before, the paper adopts SOAP message to define the service requester's usage intention. Established on the basis of XML, SOAP has good encapsulation and standardized coding rules, and at the same time, it can bind with the underlying transport protocol. Therefore, in the process of service user's submitting of the request, we firstly gain the requester's context information through the service request, and then realize contract expression of the requester's usage intention according to the SOAP message framework. Its realization procedure is as shown in Figure 5. 


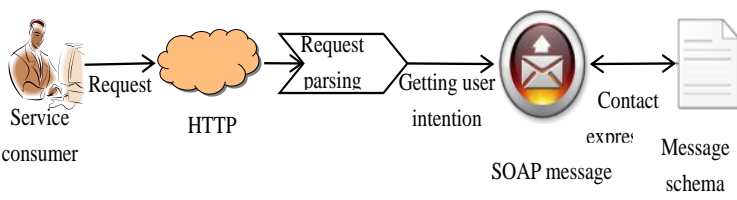

Figure5.Expression of user intention

\subsection{Pragmatic web service model based on behaviour-} intention

As mentioned before, service behavior and user's intention are the important components of pragmatics web service. For each atomic service, behavior and intention have described and expressed the pragmatic meaning of web service from the perspectives of service provider and user respectively. Although they have different emphases, they are both based on the status description and status transfer of the operational entity, which has reflected the context information of both parties of service interaction. With behavior and intention as the basis, the paper has established their projection relation and put forward the pragmatic web service model based on behavior-intention. As shown in Figure 6.

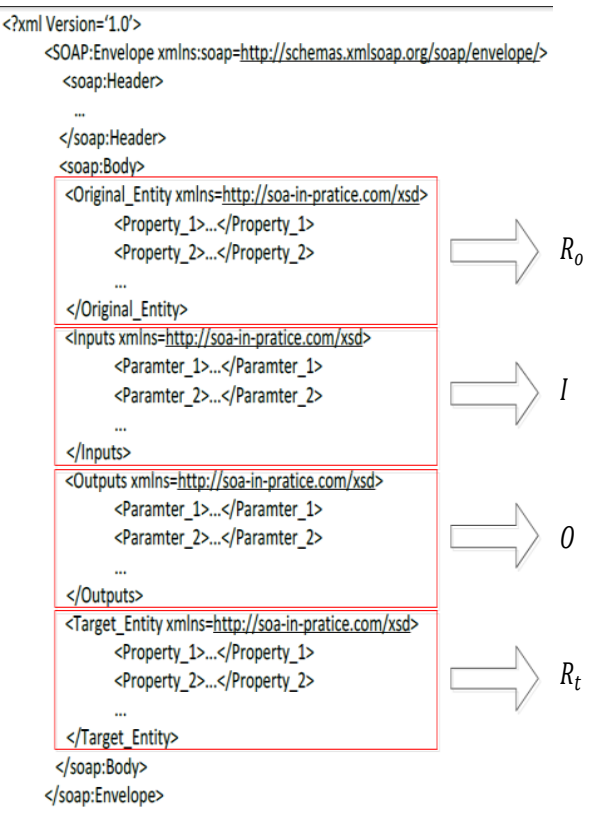

Figure 6. Example for expression of user intention based on SOAP message

From the view of structure, the pragmatic web service model is established on the basis of semantic web service, and it not only can describe the basic elements of the service objectively but also can take the context information of both parties of service interaction into account. With operational entity as the basic object, this model has achieved effective expression of the entity status and status transfer information through applicability constraint and entity attribute expansion, and it is convenient for us to classify the ground coverage information service of different types and further build their pragmatic models, which has provided precondition and basis for the service integration.

What's worth explaining is that the construction of the pragmatic web service model needs be established on the basis of the three suppositions as below:
Supposition 1: the pragmatic web service that participates in modelling should have public context knowledge, that is, operational entities of the same type can be extracted from it. Supposition 2: the pragmatic web service reflects entity's change through description and transfer of the status. Before operating the entity, the conditions that need to be met are regarded as the constraint conditions through a group of input parameters; and the result (effect and output) after the entity operation also contains a group of output parameter as the supplementary information.

Supposition 3: the description information of the service provider and the request information of the service user respectively use the same domain ontology for semantic expression in the pragmatic web service.

\section{ADAPTIVE COMPOSITION BASED ON PRAGMATIC WEB SERVICE}

Pragmatic presupposition and modelling have provided precondition and basis for the web service adaptive integration. Aiming at the issue of applicability selection and message matching among atomic services process of covering web service integration, this section has given corresponding solutions and put forward pragmatic web service on-demand assembly and message transfer and reconstruction method, and solved the issue that traditional semantic web service is unable to take hidden context information into account and it is difficult to achieve service applicability optimization and heterogeneous service system interaction. Usually, service integration always involves the combination issue of many services.

\subsection{Automatically assembly of pragmatic web services}

One of the important goals of web Service Adaptive Integration is to achieve on-demand assembly among services. On the basis of semantic description, the pragmatic web service model has given the definition of behavior-intention respectively from the perspectives of service provider and user. Based on this, we can regard the process of on-demand assembly as the matching process that whether the service behavior can satisfy users' intention, which is called "achievement". When "behavior" and "intention" operate the same entity and have a common realization goal, and the entity' applicability and input/output parameters meet certain constraint relation, the "achievement" can be reached. Therefore, we can give the following definition.

Definition 3: achievement: If one behavior $\left(\mathrm{WS}_{\text {behavior }}\right)$ and intention $\left(\mathrm{WS}_{\text {intention }}\right)$ meet the following conditions, that means "behavior realizes intention", and it is Achievement = $\left(\mathrm{WS}_{\text {behavior }}, \mathrm{WS}_{\text {intention }}\right)$.

$\mathrm{R}_{\mathrm{o}}=\mathrm{R}_{\mathrm{o}}{ }^{\prime}$, namely, behavior and intention have the same operational entity.

$\mathrm{R}_{\mathrm{t}}=\mathrm{R}_{\mathrm{t}}{ }^{\prime}$, namely, behavior and intention have the same realization goal.

$\forall \mathrm{a} \in \mathrm{A}, \forall \mathrm{ra} \in \mathrm{R}_{\mathrm{a}}$, and $\forall \mathrm{r}^{\prime} \in \mathrm{R}_{\mathrm{o}}{ }^{\prime}, \quad \operatorname{sora}(\mathrm{a})=\mathrm{r}^{\prime}$. Namely, for random applicability evaluation indicator and applicability constraint relation in $\mathrm{WS}_{\text {behavior }}$, the operational entity $\mathrm{R}_{\mathrm{o}}{ }^{\prime}$ of $\mathrm{WS}_{\text {intention }}$ has one certain attribute meeting the applicability relation.

$\mathrm{I} \in \mathrm{I}^{\prime}$ and $\mathrm{O} \in \mathrm{O}^{\prime}$, namely, the input parameter integration required by behavior is included in the input parameter integration provided by intention, and the output parameter integration of behavior includes the output parameter integration required by intention. 
According to this definition, we can build the pragmatic web service on-demand assembly model based on the agent theory, so as to solve the issue of web service applicability selection. Agent is like a closed shell in a sense, and it feels the change of the external environment through its internal symbol model and activates its internal components and makes them finish the appointed task according to the pre-customized control procedure. Its model framework is as shown in Figure 7.

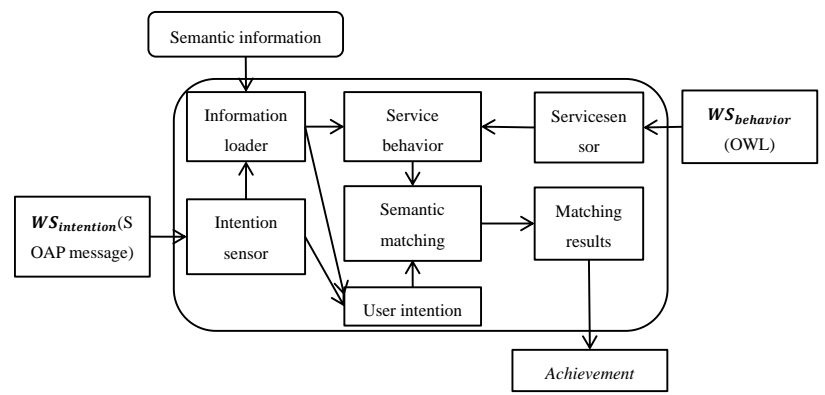

Figure. 7 Assemble model for pragmatics web service composition

It can be seen from Figure 8 that the main part of the ondemand assembly model includes 3 contents as below:

1) Sensor is in charge of extracting users' intention, service behavior and other pragmatic information from the contract expression.

2) Loader extracts pragmatic information based on domain ontology and labels the semantics on the basic contents of intention and behavior.

3) Semantic matching conducts matching from the service operational entity, target entity, inputloutput parameter and other aspects based on semantic information, and achieves service on-demand assembly according to the matching results. Figure 8 has given the example diagram of pragmatic web service matching.

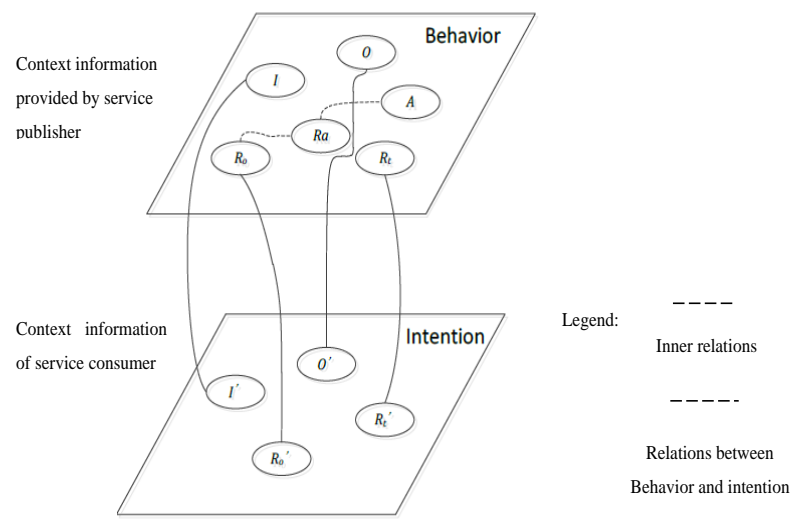

Figure8. Pragmatic web service based on Behavior-Intention model

\subsection{Pragmatic web service composition}

The pragmatic web service composition mainly consists of 3 parts: 1) One demand container used to describe demand. The container includes service demand of different types, of which the service request of the same type is divided according to certain classification mechanism. 2)A series of main (agent) engine that can be assembled as required, and they can extract the users' intention of each demand link from demand description and find the most suitable atomic service through applicability analysis and semantic matching. 3) A series of message transfer engine. According to the pre-defined message template and through the Schema matching and relation reusing, they can reconstruct the message type that users can understand, and solve the communication obstacle in the process of service combination.

In the service combination process, demand description is the precondition and basis of service combination. Based on that, atomic service can find the demand link that is suitable for participation according to its own behavior ability. Here, we use a triad to describe demand.

Definition 5:demand:Require $=\langle T, C F, W\rangle$

where $T$ : demanded switching state formed by a series of user intention $\left(W S_{\text {intention }}\right)$

$C F$ : demand switching control procedure including sequence, concurrence, selection, and other control relations. For control relations, we can use a group of symbols to express (Rong-hua et al., 2010): $C F=\{$ Sequential,Or - split, Or join, And - split, And - join $\}$.

$W$ : directed line segment pointing from the target entity $R_{t}{ }^{\prime}$ of one $W S_{\text {intention }}$ to the initial entity $R_{o}{ }^{\prime}$ of another $W S_{\text {intention. }}$.

It can be seen from definition 5 that demand description is actually the users' intention at a higher level and it consists of many atomic users' intentions and describes the operational entity's status change through their logic and control relations and the inputloutput information in this process. Figure9 has given three basic control relations between $W S_{\text {intention }}$ in the form of living examples.

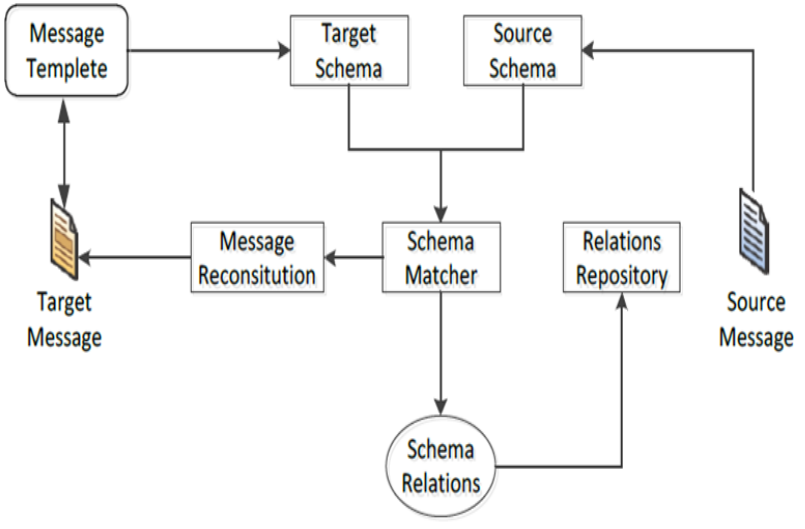

Figure 9. Process of message transformation and reconstruction

\section{CONCLUSION AND FUTURE WORKS}

GLC is one of the significant information for many fields. The integration and sharing of GLC data and processing tools are expected to promote its application. While, how we can improve the automation and intellectualization of the composition of web services is one of the problems to be solved. Current researches about service composition mainly concentrate on the semantic description of service itself and its interfaces. They can help consumers to understand the function of service deeper, but cannot treat the web services which have the same function, interface and parameters.

For GLC information service, we must composite a series of services to achieve the results. There are many algorithms that can deal with the same task, but they may have different applicability that means they may be more suitable for some images but cannot fit with other images very well. 
To solve this problem, this paper firstly analyzed syntax, semantic and pragmatic language in web service, and then introduced pragmatics web to service composition. From the viewpoint of service providers and consumers, service behavior and user intention were proposed to define and describe the composition environment. Since both behavior and intention can be formalized by the traditional service ingredients, it might be more enforceable in practice. Finally, an adaptive composition method was proposed based on the pragmatic web model. By using this method, it is easy for users to obtain the most appropriate services to fill their need.

But it should be specified that this method needs a composition template to be defined, because it need us to tell the computer the steps involved in a certain processing task. When the process takes on to a special step, this method can help users to choose the proper service. To improve this situation, domain ontology might be designed, which contains different relations among services. The service relations could make the composition process more intelligent.

\section{REFERENCES}

Castronova, A. M., Goodall, J. L., \&Elag, M. M. (2013). Models as web services using the open geospatial consortium (OGC) web processing service (WPS) standard. Environmental Modelling\& Software, 41(3), 72-83.

Cruz, S. A. B., Monteiro, A. M. V., \& Santos, R. (2012). Automated geospatial web services composition based on geodata quality requirements. Computers \& Geosciences,47(47), 60-74.

Chen, J., Chen, J., Gong, P., Liao, A.P., He, C.Y.(2011). Higher resolution global land cover mapping. Geomatics World 9 (2), 12-14 (in Chinese).

Chen, J., Wu, H., Li, S., Liao, A., He, C., \&Peng, S. (2013a). Temporal logic and operation relations based knowledge representation for land cover change web services. ISPRS Journal of Photogrammetry and Remote Sensing,83(83), 140150 .

Chen, J., Lu, M., Chen, X., Chen, J., \& Chen, L. (2013b). A spectral gradient difference based approach for land cover change detection. ISPRS Journal of Photogrammetry and Remote Sensing, 85(2), 1-12.

Chen, N., Di, L., Yu, G., \& Gong, J. (2010).Geo-processing workflow driven wildfire hot pixel detection under sensor web environment. Computers \& Geosciences, 36(3), 362-372.

Chen, X., Chen, J., Shi, Y., Yamaguchi, Y.(2012). An automated approach for updating land cover maps based on integrated change detection and classification methods. ISPRS Journal of Photogrammetry and Remote Sensing, 71 (7), 86-95.

Chen, Y., \& Ding, Y. (2008). Boundedness of commutators of marcinkiewicz integral with rough variable kernel. Integral Equations \& Operator Theory,61(4), 477-492.

Farnaghi, M., \&Mansourian, A. (2013). Disaster planning using automated composition of semantic OGC web services: a case study in sheltering. Computers Environment \& Urban Systems, 41(9), 204-218.
Feranec, J., Hazeu, G., Christensen, S., \& Jaffrain, G. (2007). Corineland cover change detection in europe (case studies of the netherlands and slovakia). Haseltonia,24(1), 234-247.

Fukatsu, T., Kiura, T., \& Hirafuji, M. (2011). A web-based sensor network system with distributed data processing approach via web application.Computer Standards \& Interfaces, 33(6), 565-573.

Gong, P., Wang, J., Yu, L., Zhao, Y., Zhao, Y., \& Liang, L., et al. (2013). Finer resolution observation and monitoring of global land cover: first mapping results with Landsat TM and ETM+ data. International Journal of Remote Sensing,34(7), $2607-2654$

Hansen, M. C., \& Loveland, T. R. (2012).A review of large area monitoring of land cover change using Landsat data. Remote Sensing of Environment,122(1), 66-74.

Herold, M., Mayaux, P., Woodcock, C. E., Baccini, A., \& Schmullius, C. (2008). Some challenges in global land cover mapping: an assessment of agreement and accuracy in existing $1 \mathrm{~km}$ datasets. Remote Sensing of Environment, 112(5), 25382556.

Verburg, P.H., Neumann, K., Nol, L. (2011). Challenges in using land use and land cover data for global change studies.Global Change Biology, 17(2), 974-989.

Xian, G., \& Homer, C. (2010).Updating the 2001 national land cover database impervious surface products to 2006 using Landsat imagery change detection methods. Remote Sensing of Environment, 114(8), 1676-1686.

Rong-Hua, Y. E., Jin, Z., Wang, P. W., Zheng, L. W., \& Yang, X. F. (2010). Approach for autonomous web service aggregation driven by requirement. Journal of Software,21(6), 1181-1195.

Sangers, J., Frasincar, F., Hogenboom, F., \& Chepegin, V. (2013).Semantic web service discovery using natural language processing techniques. Expert Systems with Applications, 40(11), 4660-4671.

Sun, Z., Yue, P., \& Di, L. (2012).Geopwtmanager: a taskoriented web geoprocessing system. Computers \& Geosciences,47(8), 34-45.

Townshend, J.R., Masek, J.G., et al.(2012). Global characterization and monitoring of forest cover using Landsat data: opportunities and challenges. International Journal of Digital Earth,5(5), 373-393.

Yue, K., Wang, X. L., \& Zhou, A. Y. (2004). Underlying techniques for web services: a survey. Journal of Software, 15(15), 428-442.

Yue, P., Gong, J., Di, L., \& He, L. (2012). Automatic geospatial metadata generation for earth science virtual data products..Geoinformatica,16(1), 1-29.

Zhai, S., Hai, W., \& Wei, J. (2008). A Pragmatics Web Service Oriented Approach to Understanding the Semantics of Concepts. Asia-Pacific Services Computing Conference, 2008.APSCC '08.IEEE (pp.1069-1074).IEEE.

Zhao, P., Foerster, T., \&Yue, P. (2012).The geoprocessing web. Computers \& Geosciences, 47(4), 3-12. 